\title{
Robust Adaptive Fuzzy Control for Planetary Rovers While Climbing up Deformable Slopes with Longitudinal Slip
}

\author{
Li Zhengcai and Wang Yang \\ School of Mechanical Instrumental Engineering, Harbin Institute of Technology, Harbin 150001, China \\ Correspondence should be addressed to Li Zhengcai; 12b908067@hit.edu.cn
}

Received 23 May 2014; Accepted 29 July 2014; Published 3 September 2014

Academic Editor: Kemao Peng

Copyright ( 2014 L. Zhengcai and W. Yang. This is an open access article distributed under the Creative Commons Attribution License, which permits unrestricted use, distribution, and reproduction in any medium, provided the original work is properly cited.

\begin{abstract}
Mobility control is one of the most essential parts of planetary rovers' research and development. The goal of this research is to let the planetary rovers be able to achieve demand of motion from upper level with satisfied control performance under the rough and deformable planetary terrain that often lead to longitudinal slip. The longitudinal slip influences the mobility efficiency obviously, especially on the major deformable slopes. Compared with the past works on normal stiff terrains, properties of soil and interaction between wheels and soil should be considered additionally. Therefore, to achieve the final goal, in this paper, wheel-soil dynamic model for six-wheel planetary rovers while climbing up deformable slopes with longitudinal slip is first built and control based in order to account for slip phenomena. These latter effects are then taken into account within terramechanics theory, relying upon nonlinear control techniques; finally, a robust adaptive fuzzy control strategy with longitudinal slip compensation is developed to reduce the effects induced by slip phenomena and modeling error. Capabilities of this control scheme are demonstrated via full scale simulations carried out with a six-wheel robot moving on sloped deformable terrain, whose real time was computed relying uniquely upon RoSTDyn, a dynamic software.
\end{abstract}

\section{Introduction}

In the field of special mobile robots environment, including planetary exploration missions, caravan survey, polar expedition, and wild fire spreading, rovers may need to traverse on deformable terrains, and the interaction between rigid wheels and soft soil has become a meaningful research topic because of longitudinal slip influence mobility control obviously [1]. In the past works on normal stiff terrains, for example, Kanayama et al. [2] proposed a stable control scheme for an autonomous mobile robot under the assumption of perfect velocity tracking. Kim and Oh [3] proposed a modified input-output linearization method to solve the problem of a decoupling matrix using a generalized inverse that provided a least-squares solution to the tracking control of two-wheeled mobile robots. Raibert et al. [4] proposed a PID controller to solve the path tracking problem of a mobile robot using a simple linearized model of the mobile robot, which was composed of an integrator and a delay. Colombano et al. [5] proposed an output-feedback controller that allowed a unicycle mobile robot to track a predefined path. However, all of these control methods based on normal stiff terrains hypothesis of nonholonomic mobile robot systems. Generally, there are two aspects concerning the control accuracy for rovers that traverse on deformable terrains. One is the modeling error. Built on terramechanics, the interaction dynamic model typically has a complicated integral and nonlinear form and must be simplified for utilization in control design. Modeling error is then unavoidable and should be kept in an acceptable range. The other is the uncertain parameters including soil parameters and mechanism parameters in the model. These soil parameters and mechanism parameters are time-variant as rover moves at various locations of deformable terrain. Possibility to deal with such problems is robust control and adaptive parameters estimation [1]. Among the system, many modern control theory techniques and stabilizing control algorithms have been proposed for the motion control of special mobile robots. The neural network control of mobile robots has been the subject of intense research in recent years $[6,7]$. Bartsch 
et al. [6] investigated tracking control using an adaptive smart neural network for robot and they produced fine motion control based on partially unknown dynamics. Bekker [8] presented a neural network-based model that combined the backstepping technique with a torque controller. For the posterior mobility control of rover, wheel-terrain interaction model is addressed. In the relevant literature, McKyes provide wheel-terrain mechanics with closed form [9]. The model describes a rigid wheel moving on deformable terrain with low cohesion as shown in Figure 4. Because the terrain is assumed as a plane, the dynamic model contains two force equations, normal stress and drawbar pull, and one driving torque equation. Such a model is decomposed by Terzaghi into three sequent formulations for the sake of convenience to identify the parameters [10]. Reece compared the simplified and original integral form by numerical analysis [11, 12]. Based on the wheel-terrain mechanics, Brunskill and Lappas, propose online soil parameter estimation using the linear least-square method [13, 14]. Janosi and Hanamoto adopt function fitting method to approximate the integrals of the original model and then Newton-Raphson method to find the solution of parameters in the nonlinear equations numerically [15-18].

However, the abovementioned models are all built on flat terrain, although deformation of terrain is considered. In practical missions, slope terrain is hard to avoid. A slopebased wheel-soil dynamic model based on control for WMR is required to be built when the rover wheels are inclined $[19,20]$. In this case, the model with two forces and one torque for flat terrain cannot fully describe the wheel-terrain dynamics on a slope. On the other hand, noticing that the previous control method, such as robust control and realtime soil parameters estimation, is strictly taking the wheelterrain dynamics model into consenter, it may cost excessive time to find the satisfactory solution. This is unnecessary and hard to be implemented.

In this paper, we develop a slope-based wheel-soil dynamic model for WMR based on a simplified interaction model and uniwheel slope-based experiment, in which the draw-bar pull can be denoted as a linear function of driving force, normal force, and slip; it is suitable for describing the rigid wheels on sloped deformable terrain. Then, the simplified slope-based wheel-soil interaction dynamic model is used for WMR slope-based control. Based on the simplified slope-based wheel-soil interaction dynamic model, control strategy can be designed simultaneously by adopting an adaptive fuzzy and slip compensation [8]. Robust control is used because the unmodeled error should be compensated timely by adaptive adjustment of the index function. The uncertain parameters include soil parameters and mechanism parameters, which make the state uncontrollable and are compensated by adaptive fuzzy strategy. The slip is the important state variable; in order to deal with this problem, we deemed as measurement model using the methods like the relevant literature. Capabilities of this control scheme is demonstrated via full scale simulations carried out with a sixwheel robot moving on sloped deformable terrain, whose real time computed relying uniquely upon RoSTDyn, a dynamic software.

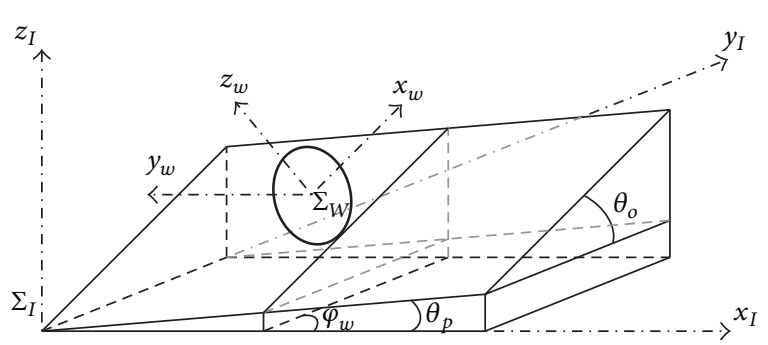

FIGURE 1: Coordinate systems of a rover wheel on sloped terrain.

This paper is organized as follows. The slope-based wheelsoil dynamic model for uniwheel is introduced in Section 2. In Section 3, the slope-based wheel-soil dynamic model for planetary rover is developed. The robust adaptive fuzzy control algorithm for planetary rover on sloped deformable terrain is established in Section 4, followed by the stability analysis in Section 5. The examples of simulations are detailed in Section 6.

\section{Slope-Based Wheel-Soil Dynamic Model for Uniwheel}

Assume that the wheels of a planetary rover are traversing perpendicularly on a sloped deformable terrain. The slope is positioned in the inertial frame $\sum_{I}\left\{x_{I}, y_{I}, z_{I}\right\}$. The local wheel frame is defined as $\sum_{W}$, where $x_{w}$ is along the forward direction, $y_{w}$ along the lateral, and $z_{w}$ the normal. $\theta_{p}, \theta_{o}$, and $\varphi_{w}$ represent the pitch, roll, and yaw angle of the wheel in the inertial coordinate system, as shown in Figure 1.

When roll angle $\theta_{o}=0^{\circ}$, the pitch angle $\theta_{p}$ makes the direction of the vertical load of the wheel deviate from that of the perpendicular stress to the slope; nevertheless, it does not violate the validity of terramechanics theory [2-4] to calculate the normal force $F_{N}$, drawbar pull $F_{\mathrm{DP}}$; and wheel driving torque $T_{R}$. Let $\theta_{1}$ and $\theta_{2}$ denote the entrance and exit angle which the wheel contact the soil; let $z_{1}$ and $z_{2}$ be the maximum and residual sinkage, respectively. Interaction between the wheel and terrain generates the normal stress $\sigma$ toward the wheel center and shearing stress $\tau$ along the wheel tangential direction at every contact point. According to the terramechanics theory [2], these two can be calculated by

$$
= \begin{cases}\left(\frac{k_{c}}{b}+k_{\phi}\right) r^{N}\left(\cos \theta-\cos \theta_{1}\right)^{N} & \left(\theta_{m} \leq \theta \leq \theta_{1}\right) \\ \left(\frac{k_{c}}{b}+k_{\phi}\right) r^{N} & \\ \times\left\{\cos \left[\theta_{1}-\frac{\theta-\theta_{2}}{\theta_{m}-\theta_{2}}\left(\theta_{1}-\theta_{m}\right)\right]\right. & \left(\theta_{2} \leq \theta<\theta_{m}\right),\end{cases}
$$




$$
\begin{aligned}
& \tau(\theta) \\
& =[c+\sigma(\theta) \tan \phi] \\
& \quad \times\left\{1-\exp \left(-\frac{r\left[\left(\theta_{2}-\theta\right)-(1-s)\left(\sin \theta_{1}-\sin \theta\right)\right]}{k}\right)\right\},
\end{aligned}
$$

where the soil sinkage exponent $N$ is a linear function of the slip ratio $s$ according to [3] and can be determined as

$$
N=n_{1}+n_{2} s
$$

in which $n_{1}$ and $n_{2}$ are real coefficients. $k_{c}$ and $k_{\varphi}$ are the cohesive modulus and frictional modulus of sinkage. $b$ denote the wheel width. The angle of maximal stress $\theta_{m}$ is expressed by

$$
\theta_{m}=\theta_{0}=\left(c_{1}+c_{2} s\right) \theta_{1} .
$$

In (2), $c$ is the internal cohesion of the soil, and $\phi$ is the friction angle; $k$ denote the shear modulus. The slip ratio in (2)-(4) is defined as

$$
s=\frac{\left(r \omega_{R}-v\right)}{r \omega_{R}}
$$

which explicates the relationship between angular rate $\omega_{R}$ and actual forward velocity $v . r$ is the radial of the wheel. However, as one can see from Figure 2, different from the case of plane terrain, the entrance and exit angles are calculated by

$$
\theta_{i}=\theta_{p}+\sin \frac{r-z_{i}}{r} ; \quad i=1,2 .
$$

For a moving wheel, there are three forces and three torques dynamic balance equations to completely describe the motion on slope. In Figure 2, it is obvious that the lateral force $F_{S}=0$, the turning torque $T_{S}$ is uncontrolled, and roll torque $T_{0}=0 . T_{0}$ is passive and uncontrollable and should be monitored for the rollover avoidance. Thus, there are three equations that can be derived: normal stress $F_{N}$, drawbar pull $F_{\mathrm{DP}}$, and driving torque $T_{R}$. From Figure 2, they have the following integral expression:

$$
\begin{gathered}
F_{N}=b\left\{\int_{\theta_{2}}^{\theta_{m}}\left[r \sigma_{2}(\theta) \cos \theta+r_{s} \tau_{2}(\theta) \sin \theta\right] d \theta\right. \\
\left.+\int_{\theta_{m}}^{\theta_{1}}\left[r \sigma_{1}(\theta) \cos \theta+r_{s} \tau_{1}(\theta) \sin \theta\right] d \theta\right\}=W, \\
F_{\mathrm{DP}}=b\left\{\int_{\theta_{2}}^{\theta_{m}}\left[r_{s} \tau_{2}(\theta) \cos \theta-r \sigma_{2}(\theta) \sin \theta\right] d \theta\right. \\
\left.+\int_{\theta_{m}}^{\theta_{1}}\left[r_{s} \tau_{1}(\theta) \cos \theta-r \sigma_{1}(\theta) \sin \theta\right] d \theta\right\}=f_{\mathrm{DP}} \\
M_{R}=r_{s}^{2} b\left[\int_{\theta_{2}}^{\theta_{m}} \tau_{2}(\theta) d \theta+\int_{\theta_{m}}^{\theta_{1}} \tau_{1}(\theta) d \theta\right]=T
\end{gathered}
$$

in which $M_{R}$ is the moment of inertia of the wheel rotational axis.

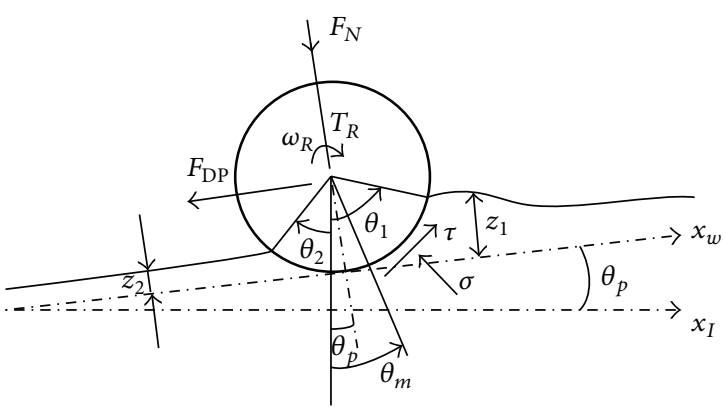

FIGURE 2: Free body diagram of a rigid wheel on deformable terrain with pitch angle.

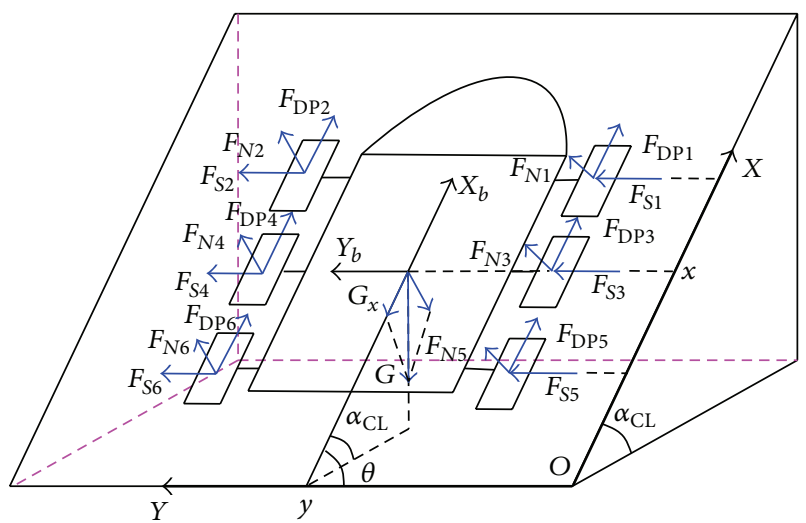

FIGURE 3: Analysis force of slope-based wheel-soil planetary rover.

\section{Slope-Based Wheel-Soil Dynamic Model for Planetary Rover}

When a planetary rover is traversing perpendicularly on a sloped deformable terrain, analysis force of slope-based wheel-soil is just like Figure 3. So the slope-based wheelsoil dynamic model was developed based on Newton-Euler method as (8)

$$
\begin{aligned}
m \ddot{x} & =\left(\sum_{i=1,3,5} F_{\mathrm{DP} i}+\sum_{i=2,4,6} F_{\mathrm{DP} i}\right)-m g \sin \alpha_{\mathrm{CL}}, \\
m \ddot{y} & =\sum_{i=1,3,5} F_{S i}+\sum_{i=2,4,6} F_{S i}, \\
I \ddot{\theta} & =\left(\sum_{i=2,4,6} F_{\mathrm{DP} i}-\sum_{i=1,3,5} F_{\mathrm{DP} i}\right) L .
\end{aligned}
$$

Due to the force $F_{\mathrm{DP}}, M_{R}$ are integrated model which is so complex that cannot be used for controller design directly; next, the integrated model should be simplified. Based on what our research had done, the force $F_{\mathrm{DP}}$ and $M_{R}$ model can be simplified as (9) and (10). Consider

$$
\begin{gathered}
\tau=F_{\mathrm{DP}} \cdot l+F_{N} \cdot e \approx F_{\mathrm{DP}} \cdot r+F_{N} \cdot e, \\
F_{\mathrm{DP}} \approx \frac{\tau}{r}-F_{N} \cdot \frac{e}{r} .
\end{gathered}
$$




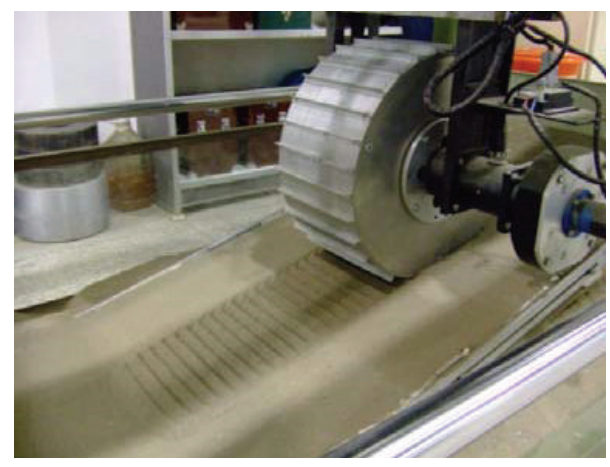

FIGURE 4: A rigid wheel on deformable terrain with pitch angle.

$F_{t}=\tau / r$ denote soil thrust caused by electrical machine drive wheel leading to soil deformation; $F_{N} \cdot e / r$ denote soil resistance, where $e / r$ is the coefficient of wheel resistance denoted by RC; RC reflects the counter of soil to wheel. The drow-pull of the wheel $F_{\mathrm{DP}}$ is the thrust subtract the soil resistance. $F_{\mathrm{DP}}$ in formula (10) and (7) are accordant, so put the formula (10) into (8), the control-based equation (11) is obtained as follows:

$$
\begin{gathered}
m \ddot{x}+\left(\mathrm{RC}_{1} \sum_{i=1,3,5} F_{N i}+\mathrm{RC}_{2} \sum_{i=2,4,6} F_{N i}\right)+m g \sin \alpha_{C L} \\
=\frac{\sum_{i=1}^{6} \tau_{i}}{r}, \\
m \ddot{y}=\sum_{i=1,3,5} F_{S i}+\sum_{i=2,4,6} F_{S i} \\
I \ddot{\theta}+\left(\mathrm{RC}_{2} \sum_{i=2,4,6} F_{N i}-\mathrm{RC}_{1} \sum_{i=1,3,5} F_{N i}\right) L \\
=\frac{\left(\sum_{i=2,4,6} \tau_{i}-\sum_{i=1,3,5} \tau_{i}\right) L}{r} .
\end{gathered}
$$

Analyzing the curve of RC, one can see from Figure 5 and Table 1 that $\mathrm{RC}$ can be donated as a linearity function, approximately, calculated by (12). The value of RC is $0.08-$ $0.32, b_{\mathrm{RC}}$ is $0.09-0.17$, and $k_{\mathrm{RC}}$ is $0.20-0.26$ approximately, from our previous experimentation. Consider

$$
\mathrm{RC}=k_{\mathrm{RC}} s+b_{\mathrm{RC}}
$$

If one define $q=\left[\begin{array}{lll}x & y & \theta\end{array}\right]^{T}$ and $\tau=\left[\begin{array}{lll}\sum_{i=1,3,5} & \tau_{i} \sum_{i=2,4,6} \tau_{i}\end{array}\right]^{T}$, from (11) and (12), the slope-based wheel-soil dynamic model for a planetary rover can be expressed as (13)

$$
\begin{gathered}
\mathbf{M}(q) \ddot{q}+\mathbf{C}(q, \dot{q}) \dot{q}+\mathbf{F}(\dot{q})+\mathbf{G}(q)+\boldsymbol{\tau}_{d} \\
=\mathbf{B}(q) \boldsymbol{\tau}+\mathbf{A}^{T}(q) \lambda,
\end{gathered}
$$

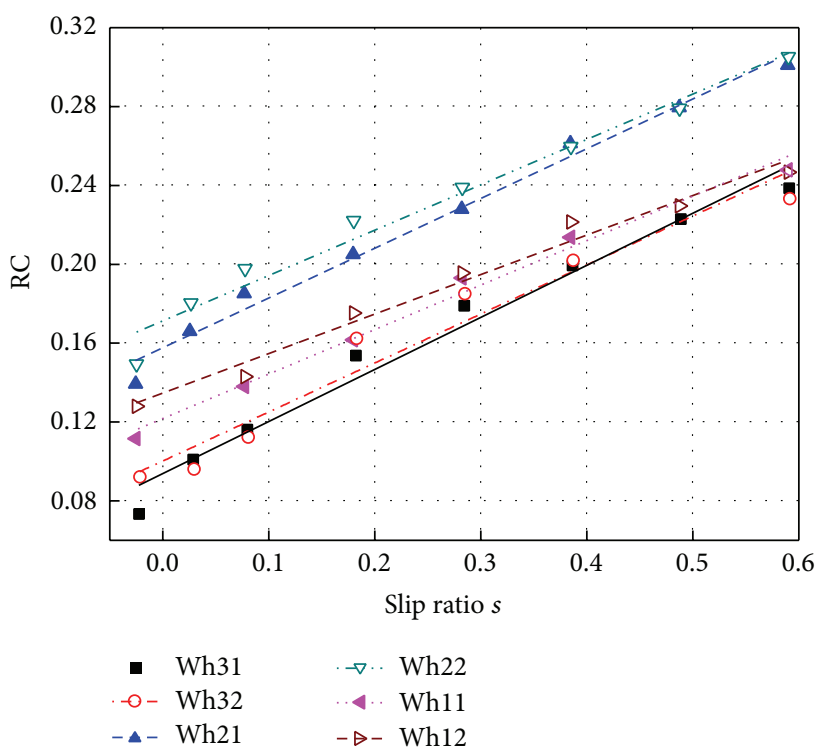

Figure 5: The value of RC and linearity curve on different soil.

TABLE 1: Resistance coefficient RC and fitting error.

\begin{tabular}{lcccc}
\hline Terrain & $b_{\mathrm{RC}}$ & Fitting error & $k_{\mathrm{RC}}$ & Fitting error \\
\hline Wh31 (soil) & 0.09378 & 0.0055 & 0.2641 & 0.0168 \\
Wh32 (silt) & 0.15759 & 0.0038 & 0.2524 & 0.0117 \\
Wh33 (snow) & 0.12167 & 0.0040 & 0.2258 & 0.0125 \\
Wh21 (field) & 0.10024 & 0.0075 & 0.2485 & 0.0252 \\
Wh21 (desert) & 0.17135 & 0.0048 & 0.2302 & 0.0148 \\
Wh21 (lunar soil) & 0.13463 & 0.0044 & 0.2006 & 0.0126 \\
\hline
\end{tabular}

where

$$
\begin{gathered}
\mathbf{M}(q)=\left[\begin{array}{ccc}
m & 0 & 0 \\
0 & m & 0 \\
0 & 0 & I
\end{array}\right], \quad \mathbf{B}(q)=\frac{1}{r}\left[\begin{array}{cc}
1 & 1 \\
0 & 0 \\
-L & L
\end{array}\right], \\
C(q, \dot{q})=\mathbf{0}, \quad \tau_{d} \leq\|H\|, \\
\mathbf{A}^{T}(q)=\left[\begin{array}{lll}
0 & 1 & 0
\end{array}\right], \quad \lambda=\sum_{i=1,3,5} F_{S i}+\sum_{i=2,4,6} F_{S i}, \\
\mathbf{G}(q)=\left[\begin{array}{c}
m g \sin \alpha_{C L} \\
0 \\
0
\end{array}\right], \\
\mathbf{F}(q, \dot{q})=\left[\begin{array}{c}
\mathrm{RC} \sum_{i=1,3,5} F_{N i}+\mathrm{RC}_{2} \sum_{i=2,4,6} F_{N i} \\
0 \\
\mathrm{RC}_{2} \sum_{i=2,4,6} F_{N i}-\mathrm{RC}_{1} \sum_{i=1,3,5} F_{N i}
\end{array}\right] .
\end{gathered}
$$

\section{Robust Adaptive Fuzzy Controller Design}

The planetary rover shown in Figure 3 is a typical example of a nonholonomic mechanical system. It has six parallel driving wheels mounted on the same axis. The motion and orientation are controlled by independent actuators; that is, 
DC motors provide the necessary torque to the six wheels. The position of the robot in an inertial Cartesian frame $\{O, X, Y\}$ is completely specified by the vector, where $x_{c}$ and $y_{c}$ are the coordinates of the center $\mathbf{C}$ of the mass and $\left\{C, X_{c}, Y_{c}\right\}$ is the local coordinate with an origin of $\left(x_{c}, y_{c}\right)$ with respect to the inertial basis. One can see from literature [] that kinematics equality constraints are independent with time and can be expressed as follows:

$$
\mathbf{A}(q) \dot{q}=0
$$

Let $\mathbf{S}(q)$ be a $n-m$ dimension and full rank matrix, formed by a set of smooth and linear independent vector fields spanning the null space of $\mathbf{A}(q)$ :

$$
\mathbf{S}^{T}(q) \mathbf{A}^{T}(q)=\mathbf{0}
$$

According to (15) and (16), it is possible to find an auxiliary vector time function $\mathbf{v}(t) \in \mathfrak{R}^{n-m}$ such that for all time $t$

$$
\dot{q}=\mathbf{S}(q) \mathbf{v}(t)
$$

Differentiating (17) yields

$$
\ddot{q}=\dot{\mathbf{S}}(q) \mathbf{v}+\mathbf{S}(q) \dot{\mathbf{v}}
$$

Then, the dynamic (13), which satisfies the nonholonomic constraint (15), can be rewritten in terms of the internal state variable $\mathbf{v}$ as

$$
\begin{aligned}
\mathbf{M}(q) & (\dot{\mathbf{S}}(q) \mathbf{v}+\mathbf{S}(q) \dot{\mathbf{v}})+\mathbf{C}(q, \dot{q}) \mathbf{S}(q) \mathbf{v} \\
& +\mathbf{F}(\dot{q})+\mathbf{G}(q)+\tau_{d} \\
= & \mathbf{B}(q) \tau+\mathbf{A}^{T}(q) \lambda
\end{aligned}
$$

By appropriate definitions one can rewrite equation as

$$
\widetilde{\mathbf{M}}(q) \dot{\mathbf{v}}+\widetilde{\mathbf{C}}(q, \dot{q}) \mathbf{v}+\widetilde{\tau}_{d}=\widetilde{\mathbf{B}}(q) \tau,
$$

where

$$
\begin{gathered}
\widetilde{\mathbf{M}}(q)=S^{T} M S, \quad \widetilde{\mathbf{C}}(q, \dot{q})=S^{T} C S+S^{T} M \dot{S}, \\
\widetilde{\tau}_{d}=S^{T}\left(\mathbf{F}(\dot{q})+\mathbf{G}(q)+\tau_{d}\right), \quad \widetilde{\mathbf{B}}=S^{T} B .
\end{gathered}
$$

We assume that the external disturbances can be expressed as

$$
\tilde{\tau}_{d} \leq\left\|S^{T} H\right\|
$$

where $H$ is a known matrix.

Exploiting the structure of the dynamic equation (20), some properties are listed as follows.

Property 1. Matrix $\widetilde{\mathbf{M}}(q)$ is symmetric and positive-definite.

Property 2. Matrix $\dot{\overrightarrow{\mathbf{M}}}(q)-2 \widetilde{\mathbf{C}}(q, \dot{q})$ is skew-symmetric.
Suppose that the desired trajectories in the task space are described by $\mathbf{q}_{d}$ and $\dot{\mathbf{q}}_{d}$. In order to track the desired trajectories, we define the sliding surface:

$$
s=\dot{e}+\sigma e,
$$

where $e=\mathbf{q}-\mathbf{q}_{d}$ is the tracking error and $\sigma$ is a constant positive definite diagonal matrix. Then define the reference state as

$$
\begin{aligned}
& \dot{q}_{r}=\dot{q}-s=\dot{q}_{d}-\sigma e, \\
& \ddot{q}_{r}=\ddot{q}-\dot{s}=\ddot{q}_{d}-\sigma \dot{e} .
\end{aligned}
$$

One now define a robust control input based on the sliding mode theory. Thus, we extend the sliding mode control law given in [15] and applied for planetary rover which is traversing perpendicularly on a sloped deformable terrain

$$
\tau=\widetilde{B}^{-1}\left(\mathbf{M}^{*} \ddot{q}_{r}+\mathbf{C}^{*} \dot{q}_{r}+\mathbf{F}^{*}-K_{s 1} s-K_{s 2} \operatorname{sign}(s)\right),
$$

where $K_{s 1}$ and $K_{s 2}$ are constant positive definite diagonal matrices. The proposed control input consists of a continuous nominal control part and a discontinuous switching control part to make the system invariant to parametric uncertainty and external disturbances. The switching control part causes the chattering problem. To reduce chattering, the switching function $\operatorname{sign}(s)$ can be replaced by a continuous approximation in the neighbourhood of the sliding surface, such as sigmoid-like function. A complete stability analysis of the sigmoid-like function is given in [17].

However, the error of the unmodel influences the performance of the proposed sliding mode controller. In the literature of $\mathrm{NN}$ control, many different models of function approximations such as Gauss function have been established to approximate the actual condition, because of their inherent approximation capabilities. The radial basis function neural network is employed for function approximation, what is assumed as a smooth function $f(x): \mathfrak{R}^{q} \rightarrow \mathfrak{R}^{2}$

$$
f(x)=\mathbf{W}^{T} \phi(x)+\varepsilon(x)
$$

where $X \in \Omega_{X} \subset \mathfrak{R}^{q}$ is the input vector, $\mathbf{W} \in$ $\mathfrak{R}^{n \times 2}$ contains the ideal thresholds $w_{10} \cdots w_{m 0}$ and weights $w_{11} \cdots w_{m L}$; and $\phi(X)=\left[\begin{array}{lllll}\phi_{1}(\theta) & \phi_{2}(\theta) & \phi_{3}(\theta) & \cdots & \phi_{n}(\theta)\end{array}\right]^{T}$ is the activation vector and $\varepsilon(\theta) \in \mathfrak{R}^{m}$ is a vector of the function approximation errors. Commonly, the Gaussian function is chosen as the activation vector

$$
\phi_{i}(X)=\exp \left[-\frac{1}{2 b_{i}^{2}}\left\|X-\xi_{i}\right\|^{2}\right], \quad i=1,2, \ldots, n,
$$

where $b_{i}$ and $\xi_{i}$ are the center and width of the $i$ th neuron.

To satisfy the universal approximation property for any continuous function $f(X): \Omega_{X} \rightarrow R^{2}$, where $\Omega_{X}$ is a compact set, there exists an estimate function such that 


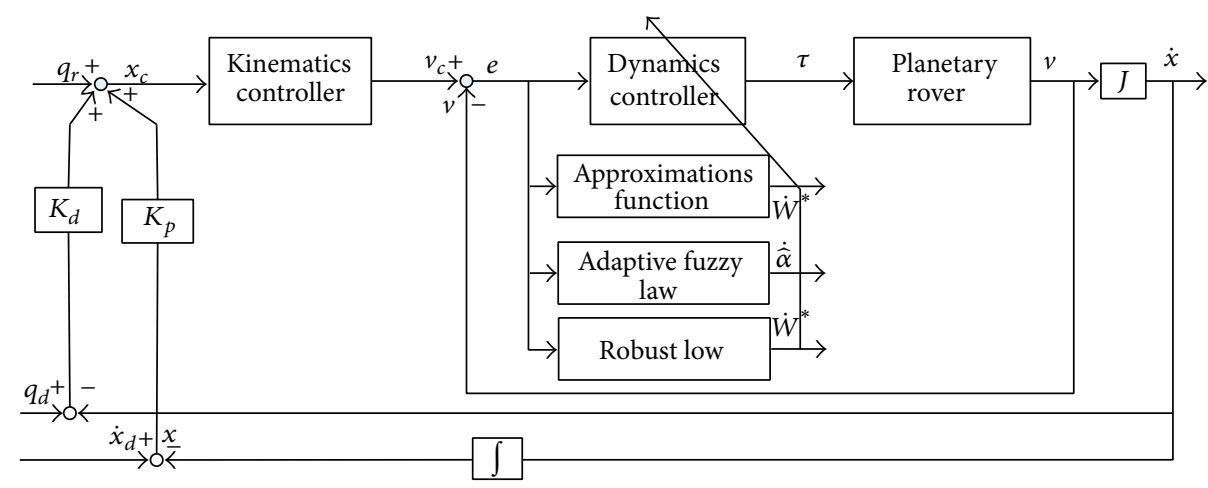

FIGURE 6: Structure of the proposed robust adaptive fuzzy motion control methodology.
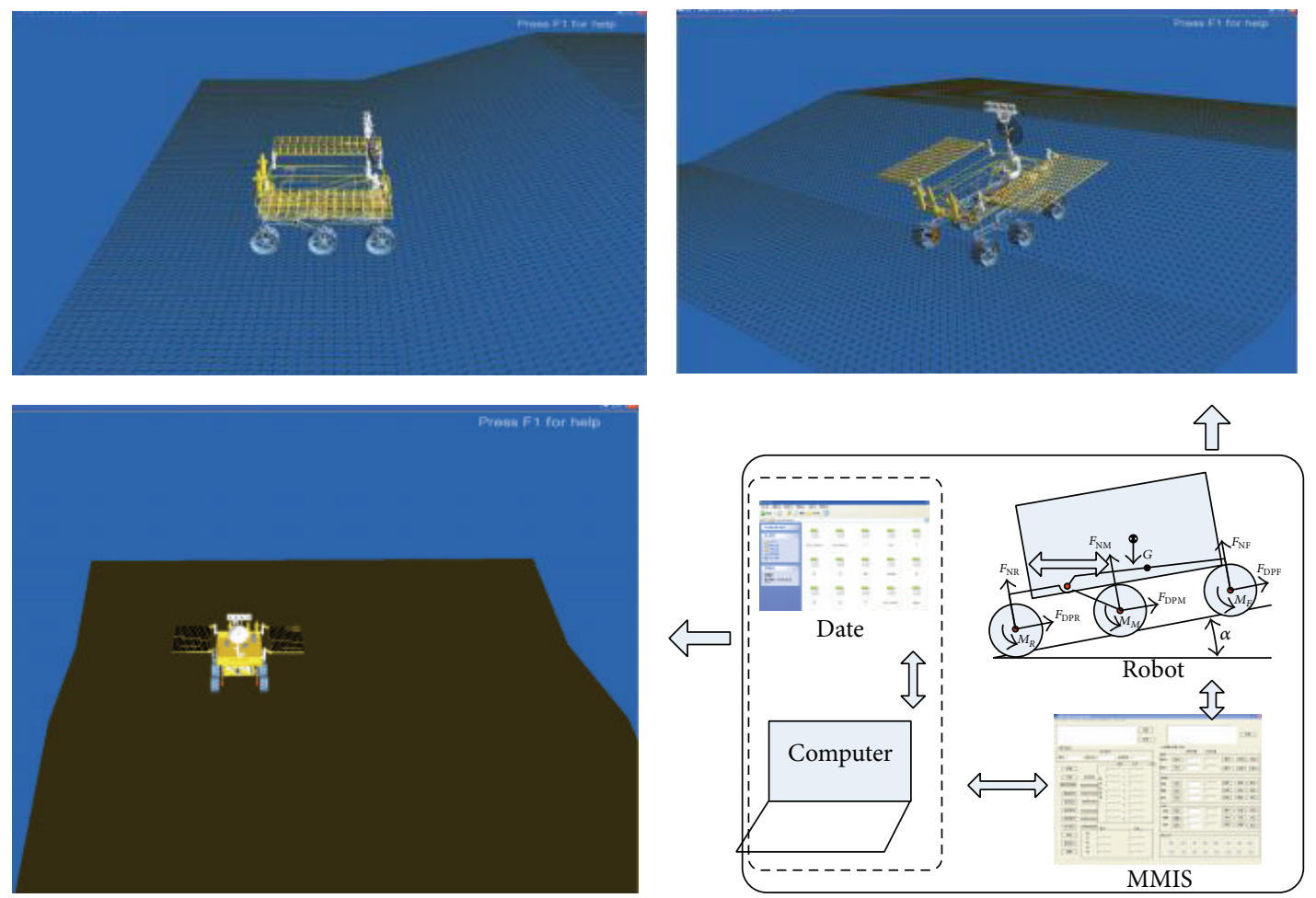

FIgURE 7: Planetary rover traverse on a slope deformable terrain simulation.

$$
f(X)=\mathbf{W}^{* T} \phi(X)+\varepsilon^{*}(X), \quad\left\|\varepsilon^{*}(X)\right\| \leq \varepsilon_{N},
$$

where $\mathbf{W}^{*}$ are optimal weights in the approximation, $\varepsilon^{*}(X)$ is the approximation error for the special case $\mathbf{W}=\mathbf{W}^{*}$, and $\varepsilon_{N}$ is a constant.

To eliminate the error influence on planetary rover traversing perpendicularly on a sloped deformable terrain, the following NN control law is considered:

$$
\tau=\widetilde{B}^{-1}\left(f(X)+\mathbf{F}^{*}-K_{s 1} s-K_{s 2} \operatorname{sign}(s)\right)
$$

in which the continuous approximation law identifier is designed as

$$
\begin{gathered}
f(X)=\mathbf{W}^{* T} \phi(X)+\varepsilon^{*}(X), \\
\dot{\mathbf{W}}^{*}=G \phi(X)\left(R^{-1} e\right)^{T},
\end{gathered}
$$

where $G$ is a positive definite matrix, and the adaptive fuzzy controller is designed as

$$
\Lambda=K_{s 2} \operatorname{sign}(s)+\varepsilon^{*}(X)
$$




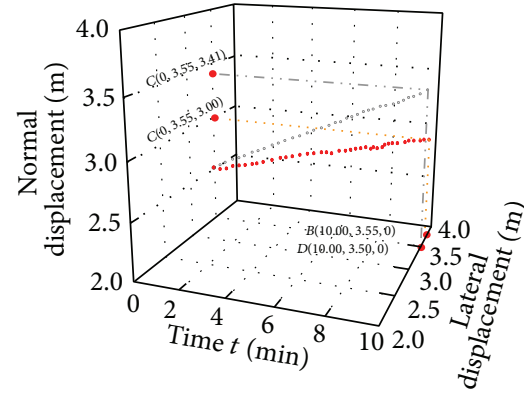

-.. Slip compensation $\star \star \star$ No slip compensation

(a)

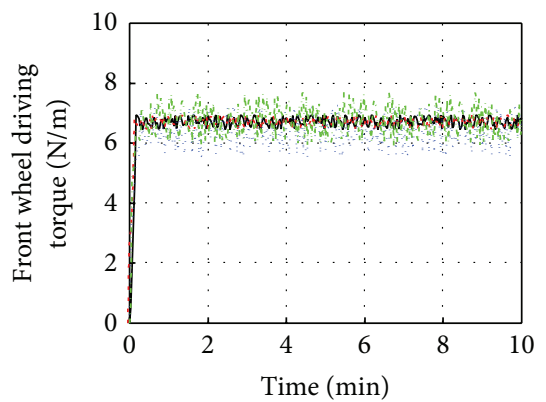

_ LF_wheel driving torque no slip control

- - - RF_wheel driving torque no slip control

.. LF_wheel driving torque with slip control

-.. RF_wheel driving torque with slip control

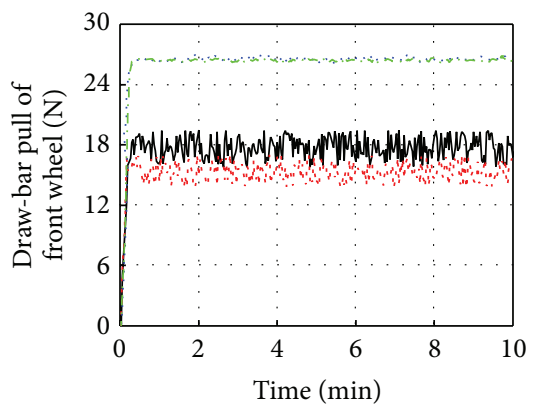

- $F_{\mathrm{DP}}$ of RF_wheel no slip control

- - $F_{\mathrm{DP}}$ of LF_wheel no slip control

$\cdots F_{\mathrm{DP}}$ of RF_wheel with slip control

-... $F_{\mathrm{DP}}$ of LF_wheel with slip control

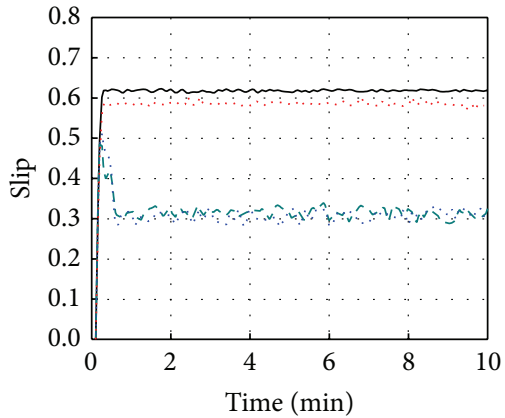

Slip of LW no control Slip of RW no control Slip of LW with control

- - - Slip of RW with control

(b)

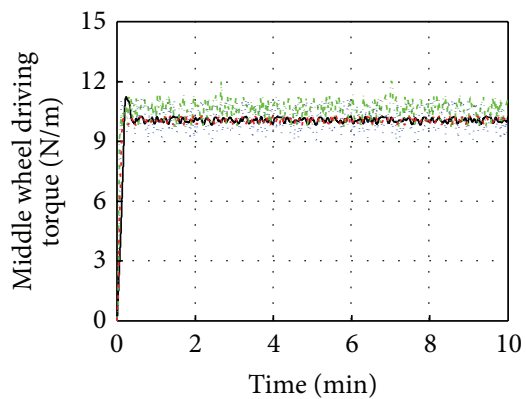

LM_wheel driving torque no slip control

- - - RM_wheel driving torque no slip control

.... LM_wheel driving torque with slip control RM_wheel driving torque with slip control

(d)

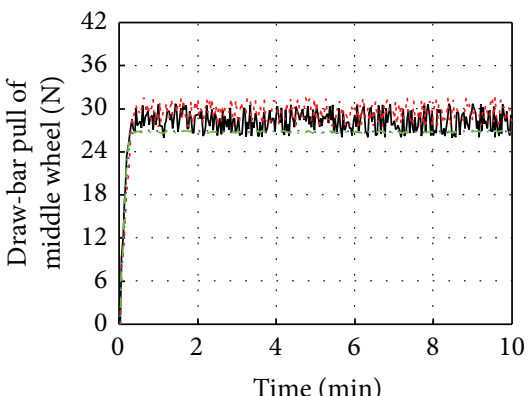

_ $F_{\mathrm{DP}}$ of RM_wheel no slip control

- - - $F_{\mathrm{DP}}$ of LM_wheel no slip control

…. $F_{\mathrm{DP}}$ of RM_wheel with slip control

_... $F_{\text {DP }}$ of LM_wheel with slip control

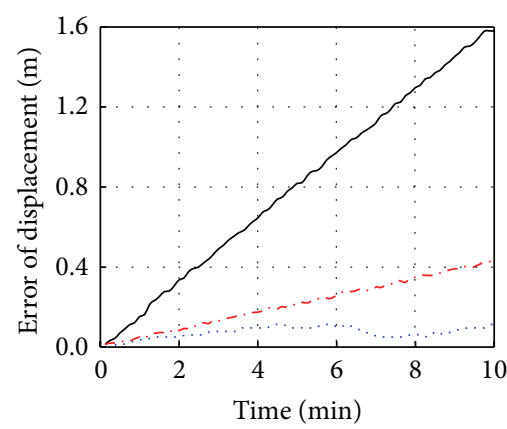

_ Error of $X$ w/o slip control

-. . Error of $Z$ w/o slip control

..... Error of $Y$ w/o slip control

(c)

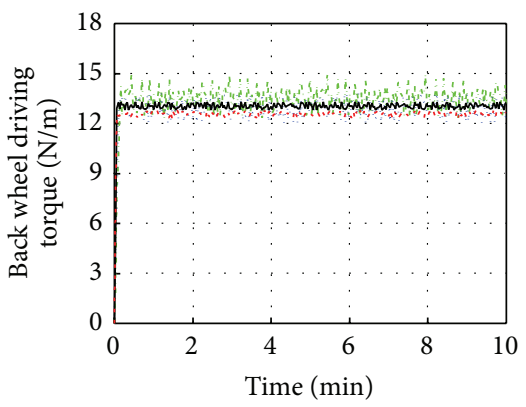

LB_wheel driving torque no slip control

- - - RB_wheel driving torque no slip control

. LB_wheel driving torque with slip control RB_wheel driving torque with slip control

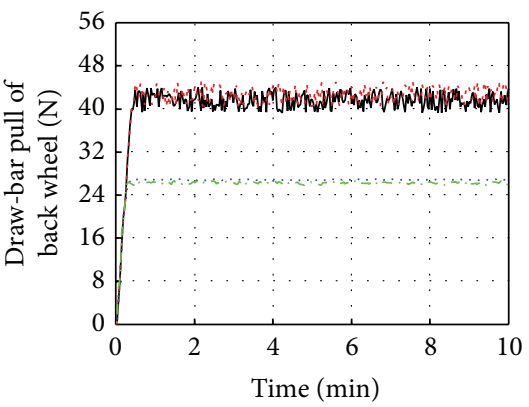

_ $F_{\mathrm{DP}}$ of RB_wheel no slip control

- - - $F_{\mathrm{DP}}$ of LB_wheel no slip control

…. $F_{\mathrm{DP}}$ of RB_wheel with slip control

_. - . $F_{\mathrm{DP}}$ of LB_wheel with slip control

(e)

FIGURE 8: A planetary rover is traversing perpendicularly on a $15^{\circ}$ slope deformable terrain. (a) Position of center of mass; (b) slip with/without control; (c) error of displacement with/without control; (d) driving torque of six wheels with/without slip control; (e) draw-bar pull of six wheels with/without slip control. 


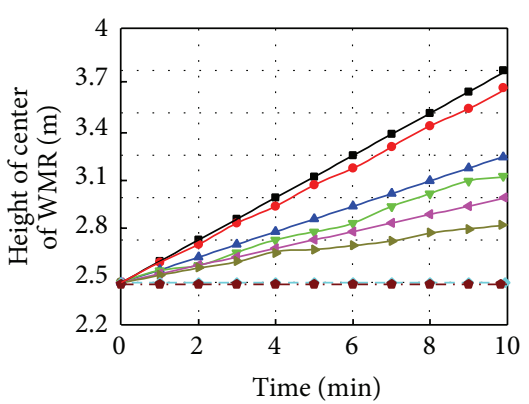

$\rightarrow-$ Height of center of WMR with slip control $25^{\circ}$ slope

$\rightarrow$ Height of center of WMR with slip control $15^{\circ}$ slope

$\neg$ Height of center of WMR no slip control $25^{\circ}$ slope

$\rightarrow$ Height of center of WMR no slip control $15^{\circ}$ slope

$\longleftarrow$ Height of center of WMR with slip control $5^{\circ}$ slope

$\rightarrow-$ Height of center of WMR no slip control $5^{\circ}$ slope

- - Height of center of WMR no slip control $0^{\circ}$ slope

- - Height of center of WMR with slip control $0{ }^{\circ}$ slope

(a)

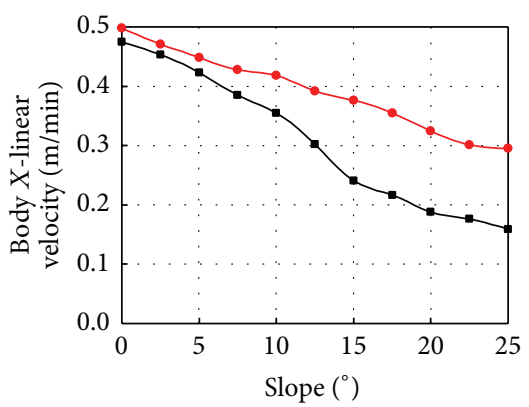

Body $X$-linear velocity no slip control

$\longrightarrow$ Body $X$-linear velocity with slip control

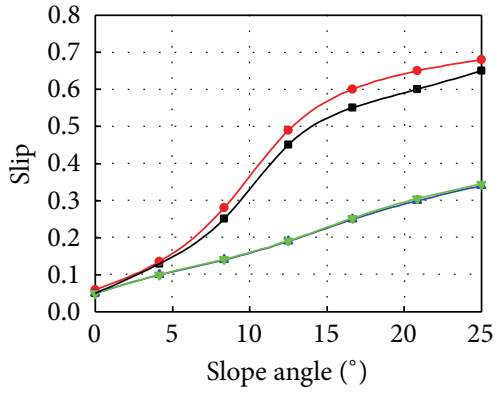

- Slip of RW no slip control

$\rightarrow$ Slip of LW no slip control

$\_$Slip of RW with slip control

$\rightarrow-$ Slip of LW with slip control

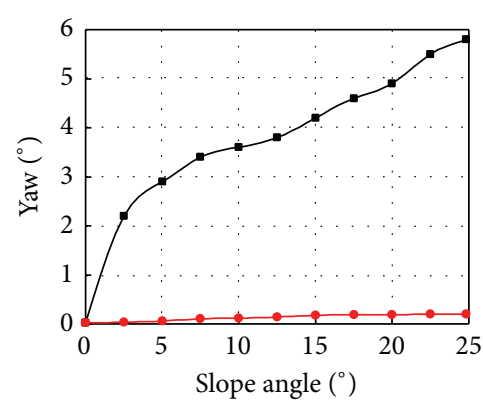

Yaw of WMR no slip control Yaw of WMR with slip control (b)

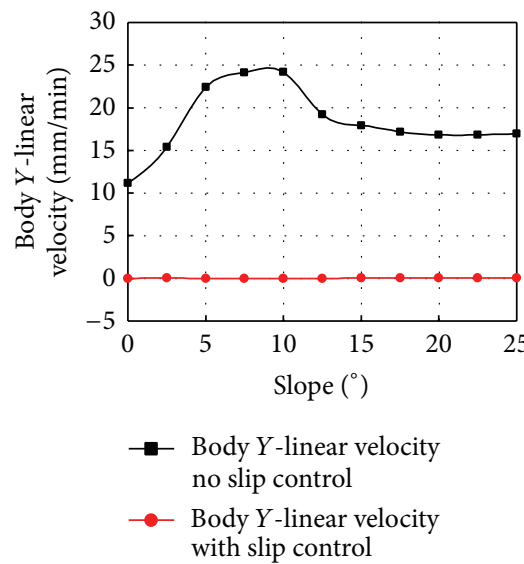

(d)

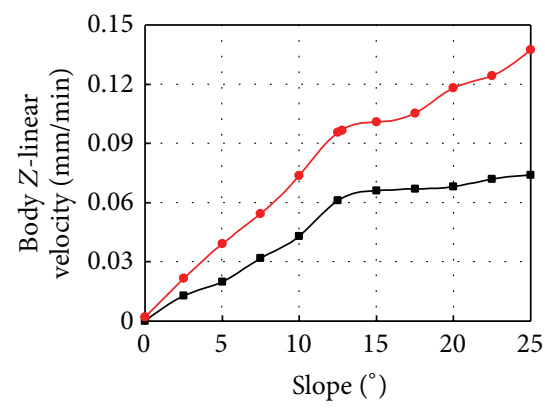

Body $Z$-linear velocity no slip control

$\rightarrow$ Body $Z$-linear velocity with slip control

FIGURE 9: A planetary rover is traversing perpendicularly on a $0^{\circ}, 8^{\circ}, 15^{\circ}$, and $18^{\circ}$ slope deformable terrain. (a) Height of center of mass; (b) slip with/without control; (c) yaw with/without control; (d) body velocity of robot with/without slip control.

with the individual fuzzy law as follows:

$$
\begin{aligned}
& \text { IF } s_{i} \text { is NB, THEN } \Lambda_{i} \text { is NB; } \\
& \text { IF } s_{i} \text { is NM, THEN } \Lambda_{i} \text { is NM; } \\
& \text { IF } s_{i} \text { is NS, THEN } \Lambda_{i} \text { is NS; } \\
& \text { IF } s_{i} \text { is ZE, THEN } \Lambda_{i} \text { is ZE; } \\
& \text { IF } s_{i} \text { is PS, THEN } \Lambda_{i} \text { is PS; } \\
& \text { IF } s_{i} \text { is PM, THEN } \Lambda_{i} \text { is PM; } \\
& \text { IF } s_{i} \text { is PB, THEN } \Lambda_{i} \text { is PB; }
\end{aligned}
$$

where NB, NM, NS, ZE, PS, PM, and PB are fuzzy sets. BS stands for negative, $\mathrm{P}$ positive, $\mathrm{B}$ big, $\mathrm{M}$ medium, $\mathrm{S}$ small, and $\mathrm{Z}$ zero; they are all Gaussian membership functions defined as follows:

$$
\mu_{A}=\exp \left[-\left(\frac{x-\alpha}{\sigma}\right)^{2}\right]
$$

$\Lambda_{i}$ can be written as

$$
\Lambda_{i}\left(x_{i}\right)=\frac{\sum_{m=1}^{M} \theta_{\Lambda_{i}}^{T} \mu_{A^{m}}\left(s_{i}\right)}{\sum_{m=1}^{M} \mu_{A^{m}}\left(s_{i}\right)}=\theta_{\Lambda_{i}}^{T} \Psi_{\Lambda_{i}}\left(s_{i}\right)
$$

in which

$$
\Psi(x)=\frac{\prod_{i=1}^{n} \mu_{A^{m}}\left(x_{i}^{*}\right)}{\sum_{m=1}^{M} \prod_{i=1}^{n} \mu_{A^{m}}\left(x_{i}^{*}\right)} .
$$

$M$ is the amount of the rules.

The configuration of the proposed robust adaptive fuzzy system, which combines a kinematics controller proposed in [], is summarized in Figure 3.

\section{Stability Analysis}

In this section, we do the system stability analysis for the closed-loop behavior of the proposed control methodology as 
shown in Figure 6. Define the reference position $\theta_{k i d}$, so that $\Lambda_{i}=\theta_{k i d}^{T} \Psi_{k i}\left(s_{i}\right)$ is the optimal compensation for $\tau$; according to Wang's theorem, there exists $\delta_{i}>0$ satisfying

$$
\left|\tau_{i}-\theta_{k i d}^{T} \Psi_{k i}\left(s_{i}\right)\right| \leq \delta_{i},
$$

where $\delta_{i}$ can be as small as possible.

Define

$$
\widetilde{\boldsymbol{\theta}}_{\Lambda_{i}}=\boldsymbol{\theta}_{\Lambda_{i}}-\boldsymbol{\theta}_{\Lambda_{i d}}
$$

Therefore, (34) can be written as follows:

$$
\Lambda_{i}=\widetilde{\theta}_{\Lambda_{i}}^{T} \Psi_{\Lambda_{i}}\left(s_{i}\right)+\theta_{\Lambda_{i d}}^{T} \Psi_{\Lambda_{i}}\left(s_{i}\right)
$$

Consider the following Lyapunov function candidate:

$$
V=\frac{1}{2} s^{T} M s+\frac{1}{2} \widetilde{W}^{T} \mu^{-1}+\frac{1}{2} \sum_{i=1}^{n}\left(\widetilde{\theta}_{\Lambda_{i}}^{T} \widetilde{\theta}_{\Lambda_{i}}\right) .
$$

Differentiating yields

$$
\begin{aligned}
& \dot{V}=\frac{1}{2} s^{T} \dot{M} s+s^{T} M \dot{s}-\widetilde{W}^{T} \mu^{-1} \dot{\widehat{W}}+\sum_{i=1}^{n}\left(\widetilde{\theta}_{\Lambda_{i}}^{T} \dot{\tilde{\theta}}_{\Lambda_{i}}\right) \\
& =\frac{1}{2} s^{T}(\dot{M}-2 C) s+s^{T}(M \dot{s}+C s) \\
& -\widetilde{W}^{T} \mu^{-1} \dot{\widehat{W}}+\sum_{i=1}^{n}\left(\widetilde{\theta}_{\Lambda_{i}}^{T} \dot{\tilde{\theta}}_{\Lambda_{i}}\right) \\
& =-s^{T} A s+s^{T}(\tau-\Lambda)+\sum_{i=1}^{n}\left(\widetilde{\theta}_{\Lambda_{i}}^{T} \dot{\tilde{\theta}}_{\Lambda_{i}}\right) \\
& =-s^{T} A s+\sum_{i=1}^{n} s_{i}\left(\tau_{i}-\Lambda_{i}\right)+\sum_{i=1}^{n}\left(\widetilde{\theta}_{\Lambda_{i}}^{T} \dot{\tilde{\theta}}_{\Lambda_{i}}\right) \\
& =-s^{T} A s+\sum_{i=1}^{n} s_{i}\left(\tau_{i}-\left(\widetilde{\theta}_{\Lambda_{i}}^{T} \Psi_{\Lambda_{i}}\left(s_{i}\right)+\theta_{\Lambda_{i d}}^{T} \Psi_{\Lambda_{i}}\left(s_{i}\right)\right)\right) \\
& +\sum_{i=1}^{n}\left(\widetilde{\theta}_{\Lambda_{i}}^{T} \dot{\widetilde{\theta}}_{\Lambda_{i}}\right) \\
& =-s^{T} A s+\sum_{i=1}^{n} s_{i}\left(\tau_{i}-\theta_{\Lambda_{i d}}^{T} \Psi_{\Lambda_{i}}\left(s_{i}\right)\right) \\
& +\sum_{i=1}^{n}\left(\widetilde{\theta}_{\Lambda_{i}}^{T} \dot{\tilde{\theta}}_{\Lambda_{i}}-\widetilde{\theta}_{\Lambda_{i}}^{T} \Psi_{\Lambda_{i}}\left(s_{i}\right)\right) \\
& =-s^{T} A s+\sum_{i=1}^{n} s_{i}\left(\tau_{i}-\theta_{\Lambda_{i d}}^{T} \Psi_{\Lambda_{i}}\left(s_{i}\right)\right) \\
& +\sum_{i=1}^{n} \widetilde{\theta}_{\Lambda_{i}}^{T}\left(\dot{\tilde{\theta}}_{\Lambda_{i}}-s_{i} \Psi_{\Lambda_{i}}\left(s_{i}\right)\right) \\
& =-s^{T} A s+\sum_{i=1}^{n} s_{i}\left(\tau_{i}-\theta_{\Lambda_{i d}}^{T} \Psi_{\Lambda_{i}}\left(s_{i}\right)\right),
\end{aligned}
$$

where

$$
\dot{\tilde{\theta}}_{\Lambda_{i}}=s_{i} \Psi_{\Lambda_{i}}\left(s_{i}\right)
$$

Assume

$$
\left|\tau_{i}-\theta_{\Lambda_{i d}}^{T} \Psi_{\Lambda_{i}}\left(s_{i}\right)\right| \leq w_{i} \leq \gamma_{i}\left|s_{i}\right|
$$

where $0 \leq\|\gamma\| \leq 1$; then

$$
s_{i}\left(\tau_{i}-\theta_{\Lambda_{i d}}^{T} \Psi_{\Lambda_{i}}\left(s_{i}\right)\right) \leq \gamma_{i}\left|s_{i}\right|^{2}=\gamma_{i} s_{i}^{2} .
$$

Putting (42) into (40), we have

$$
\begin{aligned}
\dot{V} & \leq-s^{T} A s+\sum_{i=1}^{n} \gamma_{i} s_{i}^{2}=-\sum_{i=1}^{n} a_{i} s_{i}^{2}+\sum_{i=1}^{n} \gamma_{i} s_{i}^{2} \\
& =\sum_{i=1}^{n}\left(\gamma_{i} s_{i}^{2}-a_{i} s_{i}^{2}\right)=\sum_{i=1}^{n}\left(\gamma_{i}-a_{i}\right) s_{i}^{2} \leq 0,
\end{aligned}
$$

where $\gamma=\operatorname{diag}\left[\gamma_{1}, \ldots, \gamma_{i}, \ldots, \gamma_{n}\right], a_{i}>\gamma_{i} . V \geq 0, \dot{V} \leq 0$ are guaranteed negative and this shows that $V \rightarrow 0$ and implies $e_{c} \rightarrow 0$ and $\dot{e}_{c} \rightarrow 0$ as $t \rightarrow \infty$. Furthermore, (28) shows $\dot{V}=0$, if and only if $s=0$. Therefore, global stability is guaranteed by the Lyapunov theorem. Both the system stability and velocity tracking convergence are guaranteed by control law (29), (31), and (32) driving the system (40) to closely track the desired motion trajectories.

\section{Simulation Results}

When a planetary rover is traversing perpendicularly on a sloped deformable terrain as shown in Figure 7, slippage occurs between the wheels and the ground, and most of the wheel velocities are influenced by the slip ratio. This section, the robust adaptive fuzzy control strategy with slip compensation designed upside on this paper, is demonstrated via full scale experiments carried out with the six-wheel robot moving on sloped deformable terrain. The feedback method of slip ratios is acquisition from [11]. Therefore, we assume that the reference trajectory is a line, where $x_{r}=t / 2$, $y_{r}=3.5$, and $t \in[0,10]$ and where the initial position is $Q=(0,3.5,2.5)$. The slope of the experimentation working condition is set as $0^{\circ}, 8^{\circ}, 15^{\circ}$, and $18^{\circ}$. The linear speed of planetary rover is set as $v=0.5 \mathrm{~m} / \mathrm{min}$.

When a planetary rover is traversing perpendicularly on a $15^{\circ}$ slope deformable terrain, the comparison of capabilities with/without wheel-soil dynamic model is denoted by Figure 8; form the capabilities one can see that the longitudinal slip influences the planetary rover mobility obviously.

The response of this controller is shown in Figure 9. We compensated for lost velocity due to wheel slippage. It is obvious that the tracking errors were almost the same as $A$. The results show that slip-compensation plays a significant role. Thus, when slippage occurred between the wheels and the ground, the control method with slip-compensation and adaptive NN control laws performed well during tracking control. 


\section{Conflict of Interests}

The authors declare that there is no conflict of interests regarding the publication of this paper.

\section{Acknowledgments}

This work was supported by the National Natural Science Foundation of China (50975059/61005080), China Postdoctoral Science Foundation (20100480994), and Special Foundation (201104431) and the "111" Project (B07018).

\section{References}

[1] NASA Mars Science Laboratory Factsheet, http://www.nasa.gov/ centers/goddard/news/topstory/2008/radiometer_delivery.html.

[2] European Space Agency, ExoMars Science Management Plan: Doc. No: EXM-MS-PL-ESA-00002, 12th February 2010.

[3] Google Lunar X Competition Rules, 2011, http://www. googlelunarxprize.org/les/downloads/lunar/GLXP Guidelines v3 Nov 20 2008.pdf.

[4] M. Raibert, K. Blankespoor, G. Nelson, and R. Playt, "BigDog, the rough-terrain quaduped robot," in Proceedings of the17th World Congress: The International Federation of Automatic Control, Seoul, Korea, July 2008.

[5] S. Colombano, F. Kirchner, and D. Spenneberg, "Exploration of planetary terrains with a legged robot as a scout adjunct to a rover," in Proceedings of the Institute of Aeronautics and Astronautics, Space Conference, San Diego, Calif, USA, 2004.

[6] S. Bartsch, T. Birnschein, F. Cordes et al., "SpaceClimber: development of a six-legged climbing robot for space exploration," in Proceedings of the 41st International Symposium on Robotics, pp. 1265-1272, June 2010.

[7] G. P. Scott and C. M. Saaj, "Measuring and simulating the effect of variations in soil properties on microrover tracability," in Proceedings of the SPACE Conference, American Institute of Aeronautics and Astronautics, Pasadena, Calif, USA, 2009.

[8] M. Bekker, Introduction to Terrain Vehicle Systems, Part 1-The Terrain \& Part 2-The Vehicle, vol. 2, University of Michigan Press, Ann Arbor, Mich, USA, 1959.

[9] E. McKyes, Soil Cutting and Tillage, Elsevier Science, Amsterdam, The Netherlands, 1985.

[10] K. Terzaghi, Theoretical Soil Mechanics, John Wiley \& Sons, London, UK, 3rd edition, 1943.

[11] A. R. Reece, "The fundamental equation of earth-moving mechanics," Proceedings of the Institution of Mechanical Engineers, Conference Proceedings, vol. 179, no. 6, pp. 16-22, 1964.

[12] R. D. Grisso and J. V. Perumpral, "A soil tool interaction model for narrow tillage tools," American Society of Agricultural Engineers (ASAE), 1980.

[13] C. Brunskill and V. Lappas, "The effect of relative soil density on microrover tra-cability under low ground pressure conditions," in Prceedings of the 11th European Regional Conference of the International Society for Terrain-Vehicle Systems, Bremen, Germany, 2009.

[14] J. Y. Wong, Theory of Ground Vehicles, John Wiley \& Sons, New York, NY, USA, 4th edition, 2008.

[15] Z. Janosi and B. Hanamoto, "The analytical determination of drawbar pull as a function of slip for tracked vehicles in deformable soils," in Proceedings of the ISTVS 1st International
Conference on Mechanics of Soil-Vehicle Systems, pp. 707-736, Edizioni Minerva Tecnica, Torino, Italy, 1961.

[16] R. Godbole and R. Alcock, "A device for the in situ determination of soil deformation modulus," Journal of Terramechanics, vol. 32, no. 4, pp. 199-204, 1995.

[17] A. R. Reece, Problems of Soil-Vehicle Mechanics, US Army Land Locomotion Lab, ATAC, Warren, Mich, USA, 1964.

[18] L. Richter, A. Ellery, Y. Gao, S. Michaud, N. Schmitz, and S. Weiss, A Predictive Wheel-Soil Interaction Model for Planetary Rovers Validated in Testbeds and Against MER Mars Rover Performance Data, Budapest, Hungary, 2006.

[19] M. Lyasko, "Slip sinkage effect in soil-vehicle mechanics," Journal of Terramechanics, vol. 47, no. 1, pp. 21-31, 2010.

[20] A. Ellery, "Environment-robot interaction-the basis for mobility in planetary micro-rovers," Robotics and Autonomous Systems, vol. 51, no. 1, pp. 29-39, 2005. 

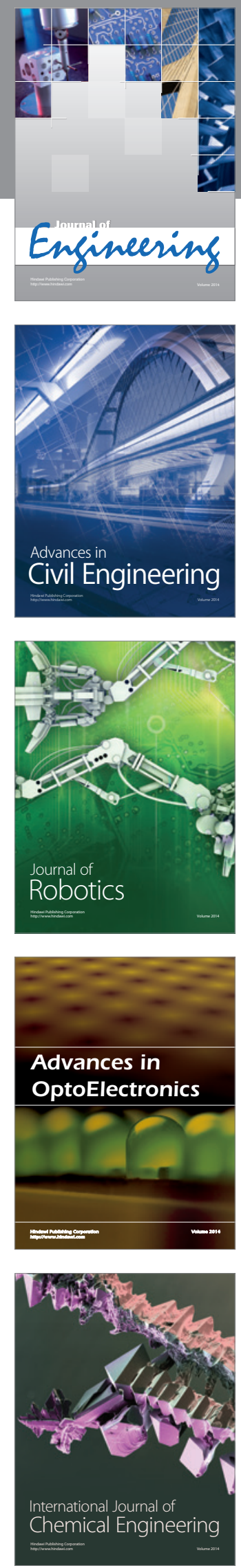

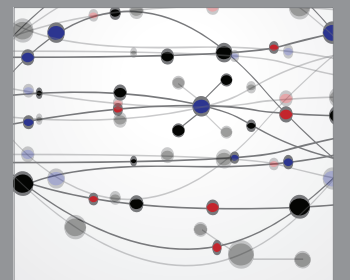

The Scientific World Journal
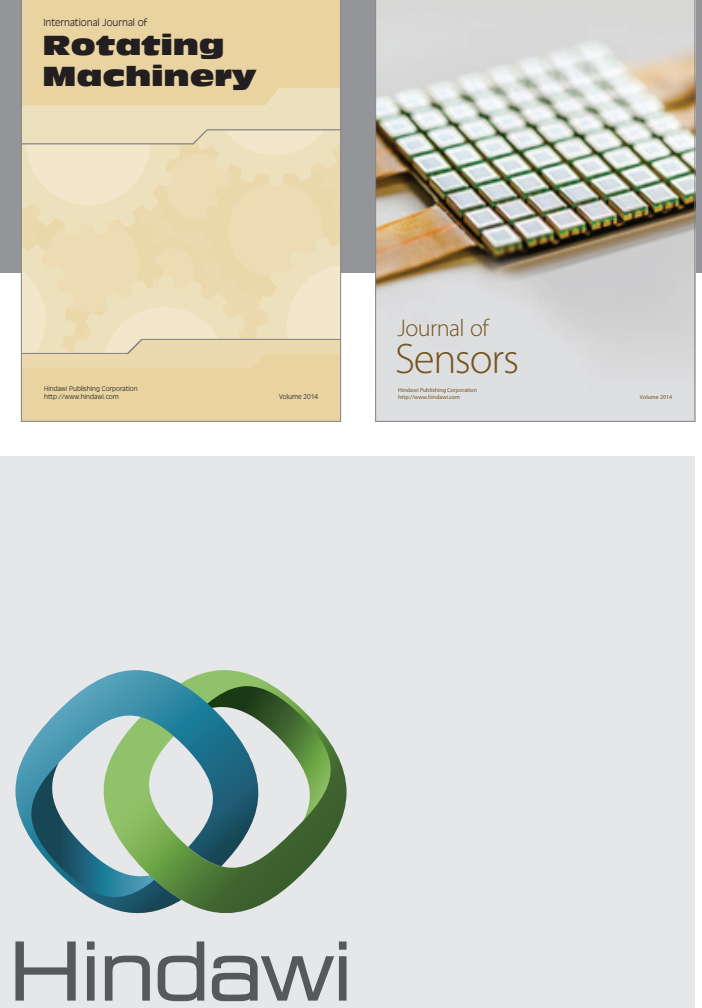

Submit your manuscripts at http://www.hindawi.com
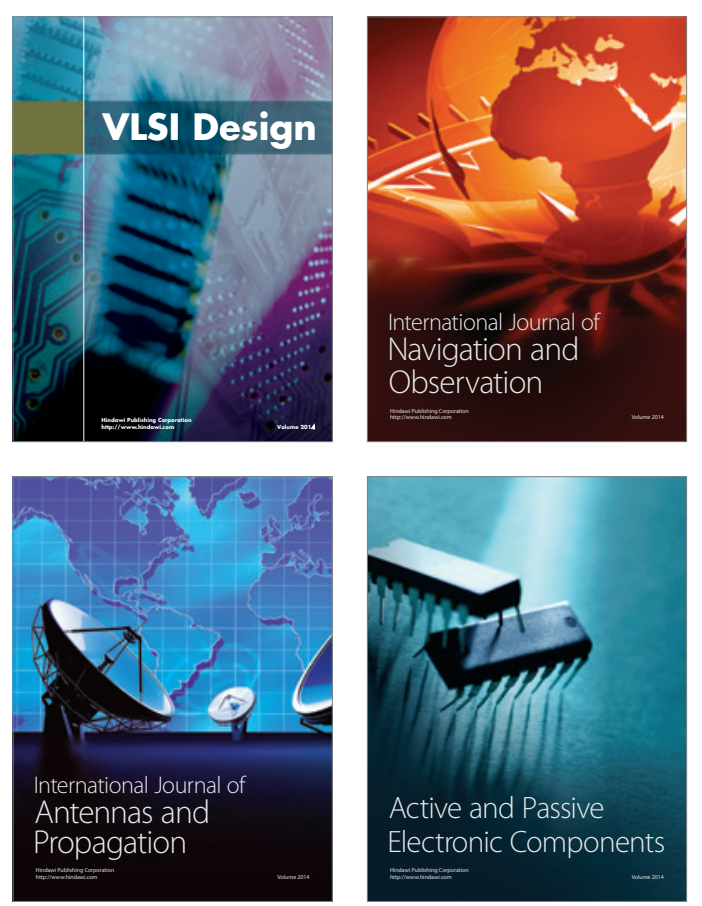
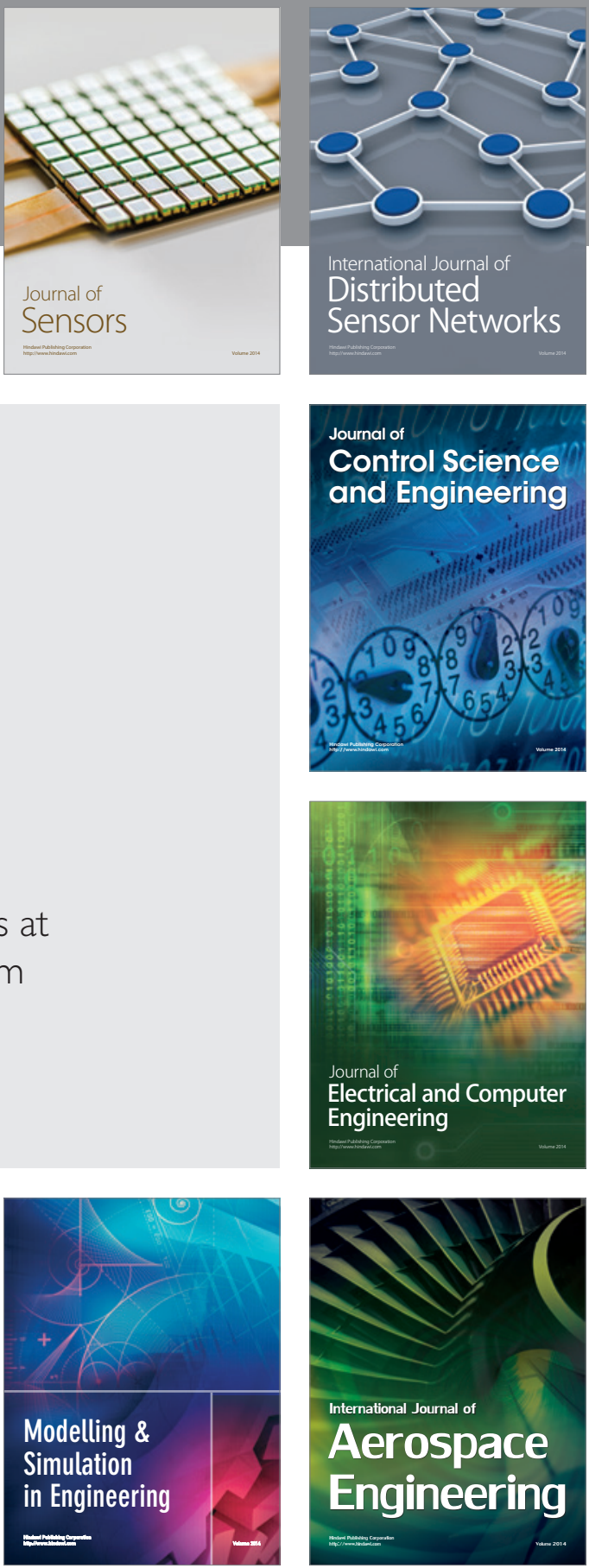

Journal of

Control Science

and Engineering
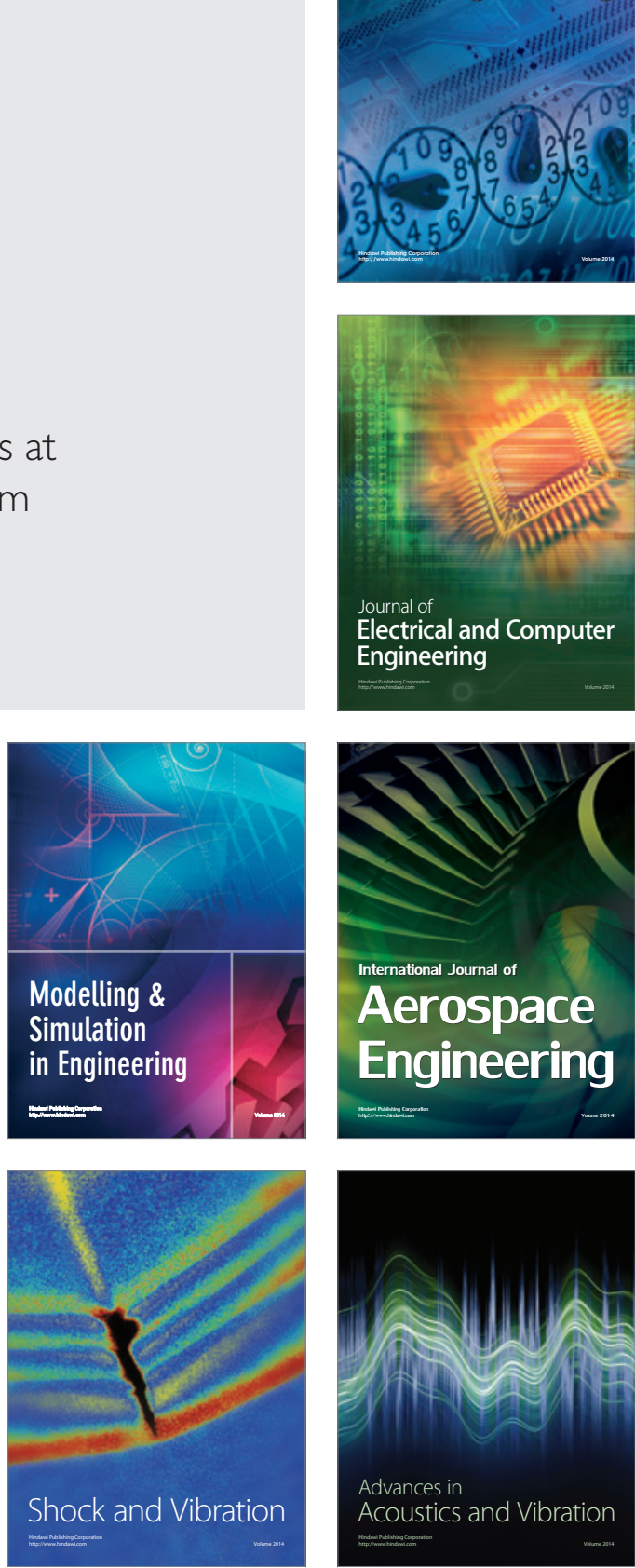